\title{
M15, an astrometric standard for CCD observations ${ }^{\star}$
}

\author{
J.-F. Le Campion ${ }^{1}$, J. Colin ${ }^{1}$ and M. Geffert ${ }^{1,2}$ \\ 1 Observatoire de Bordeaux, URA 352, CNRS/INSU, F-33270 Floirac, France \\ 2 Sternwarte der Universität Bonn, Auf dem Hügel 71, 53121 Bonn, Germany
}

Received May 4, 1995; accepted March 7, 1996

\begin{abstract}
CCD observations of stars in the region of the globular cluster M15 have been used to obtain astrometric positions of 1092 stars in the central part of the cluster within the FK5 system. The purpose of this catalogue is to calibrate astrometrically CCD observations by means of measuring the positions of stars in M15. The comparison with other catalogues and with measurements of photographic plates of the central part of M15 indicates a mean accuracy of about 50 to 70 mas for each star of our catalogue.
\end{abstract}

Key words: astrometry — globular clusters: M15

\section{Introduction}

Until now CCD observations were mainly used for photometry, spectroscopy and imaging. However, CCDs measurements have also successfully been applied in narrow field astrometry first for parallaxes (Monet \& Dahn 1983), later for the determination of positions of double stars (e.g. Sinachopoulos \& Seggewiß 1990) and for the determination of positions of X-ray candidates in the centre of globular clusters (Geffert et al. 1994). More recently the equipment of meridian circles with CCD detectors (e.g. Requième et al. 1994) yields very promising results. An additional possible astrometric application of this technique may be the determination of positions of fast moving objects like asteroids or satellites around planets since the exposure times with CCD's are shorter than with photographic plates. In every case it is necessary to calibrate the telescope-detector combination in order to convert the rectangular positions on the frame into spherical ones. This calibration can be realised by a sufficient number of coordinates of reference stars on the frame. Another possibility are astrometric calibration frames, which were taken before and after the observations. First attempts to provide suited reference fields have been undertaken by Geffert et al. (1993a) and Sinachopoulos et al. (1994). Preferentially these reference fields must contain in a small region many stars with known proper motions, or with mainly the same proper motion for all stars. Moreover, the stars must have a large range of magnitudes in order to be usable for different research subjects, requiring dif-

Send offprint requests to: J. Colin

* Table 1 is only available in electronic form at the CDS via anonymous ftp 130.79.128.5 ferent limiting magnitudes. One of the best candidates are the globular clusters which satisfy most of the constraints mentioned above.

We have chosen M15 for several reasons: a) We have recently made a catalogue of positions and proper motions in the system of the FK5 for about 1000 stars for a field of $6 \times 8$ square degree around this cluster (Le Campion et al. 1992a). This catalogue is very suited for the reduction of further material covering a few arcminutes around the cluster.

b) The absolute proper motion of the cluster by the use of plates from Bordeaux, Bonn, CERGA, and glass copies of the Palomar Sky Survey has been recently determined (Geffert et al. 1993b).

c) Saturn was located near M15 in the sky, when the first mutual phenomena of its satellites were observed at the end 1994.

d) Intensive efforts have been undertaken to study the core of this cluster, which is probably the best candidate for a cluster with core collapse. Based on observations of the HST also a significant number of blue stragglers, important tracers of the dynamical evolution of star clusters, have been found (Lauer et al. 1991; De Marchi \& Paresce 1994; Stetson 1994; Yanny et al. 1994).

e) Optical identification of the X-ray source and the pulsars in the centre of M15 is also an important question (Aurière et al. 1984; Prince et al. 1991; Taylor et al. 1993). For the identifications of optical counterparts it is important to have the positions in the FK5 system, which is in our case represented by the reduction of our data with respect to the catalogue of Le Campion et al. (1992a).

Earlier catalogues of Küstner (1921), Buonano et al. (1983) with its extension of Battistini et al. (1985), and 
Table 1. The catalogue of positions of stars in a $8^{\prime} \times 6^{\prime}$ region of the globular cluster M15. Designations are taken from Küstner $1921(\mathrm{Ku})$, Buonanno et al. 1983 (Buon) or internal numbers. NOA and NOD are the number of observations for the determination of $\alpha$ and $\delta$. The mean epoch of the catalogue is 1992, while the positions were determined with respect to the catalogue of Le Campion et al. (1992a). Since this catalogue was reduced to the PPM, our positions were determined with respect to the FK5 system

\begin{tabular}{|c|c|c|c|c|c|c|}
\hline Star 1 & $\begin{array}{l}\alpha_{\mathrm{J} 2000} \\
{[\mathrm{~h} \mathrm{~m} \mathrm{~s}]}\end{array}$ & $\begin{array}{l}\delta_{\mathrm{J} 2000} \\
{\left[\begin{array}{lll}0 & \prime & \prime \prime\end{array}\right]}\end{array}$ & $\begin{array}{r}\sigma_{\alpha} \\
{[\mathrm{mas}]}\end{array}$ & $\begin{array}{r}\sigma_{\delta} \\
{[\mathrm{mas}]}\end{array}$ & $\mathrm{NOA}$ & NOD \\
\hline 1 & 212958.120 & +121001.16 & 20 & 50 & 5 & 5 \\
\hline 2 & 212957.830 & +121002.29 & 90 & 80 & 6 & 6 \\
\hline 3 & 212957.982 & +120958.13 & 70 & 100 & 3 & 3 \\
\hline 4 & 212958.079 & +120957.13 & 20 & 60 & 2 & 3 \\
\hline 5 & 212957.736 & +121001.98 & 60 & 40 & 3 & 3 \\
\hline 6 & 212958.018 & +120956.84 & 130 & 70 & 7 & 4 \\
\hline 7 & 212958.004 & +121005.52 & 30 & 30 & 6 & 6 \\
\hline 8 & 212957.678 & +121000.13 & 90 & 110 & 6 & 6 \\
\hline 9 & 212958.343 & +121000.60 & 00 & 150 & 1 & 3 \\
\hline 10 & 212957.639 & +121004.28 & 210 & 120 & 2 & 2 \\
\hline 11 & 212957.839 & +121007.24 & 40 & 80 & 6 & 6 \\
\hline 12 & 212958.451 & +121000.39 & 20 & 170 & 2 & 2 \\
\hline 13 & 212958.173 & +121007.48 & 50 & 50 & 5 & 5 \\
\hline 14 & 212957.543 & +121002.58 & 120 & 40 & 4 & 4 \\
\hline 15 & 212958.359 & +121006.58 & 50 & 120 & 3 & 3 \\
\hline Ku539 & 212957.902 & +120952.96 & 60 & 60 & 15 & 15 \\
\hline 16 & 212957.606 & +120955.08 & 50 & 50 & 15 & 15 \\
\hline 17 & 212957.668 & +121007.79 & 90 & 90 & 3 & 3 \\
\hline 18 & 212958.265 & +120953.47 & 160 & 100 & 3 & 3 \\
\hline 19 & 212958.291 & +121008.46 & 20 & 130 & 3 & 3 \\
\hline 20 & 212958.631 & +121002.51 & 60 & 10 & 4 & 4 \\
\hline 21 & 212958.015 & +121010.72 & 40 & 30 & 6 & 6 \\
\hline 22 & 212957.382 & +121004.43 & 110 & 100 & 6 & 6 \\
\hline 23 & 212957.390 & +120956.71 & 130 & 80 & 3 & 3 \\
\hline 24 & 212958.434 & +121009.03 & 20 & 130 & 3 & 3 \\
\hline 25 & 212957.794 & +121011.05 & 120 & 60 & 6 & 6 \\
\hline 26 & 212958.156 & +121011.32 & 40 & 140 & 3 & 4 \\
\hline 27 & 212958.716 & +121002.06 & 120 & 80 & 4 & 4 \\
\hline 28 & 212958.665 & +120956.56 & 130 & 50 & 8 & 8 \\
\hline 29 & 212958.411 & +120951.54 & 90 & 50 & 5 & 4 \\
\hline 30 & 212957.410 & +120953.86 & 40 & 40 & 14 & 14 \\
\hline 31 & 212957.226 & +121000.29 & 70 & 90 & 6 & 5 \\
\hline 32 & 212958.622 & +121007.99 & 40 & 80 & 4 & 4 \\
\hline 33 & 212958.284 & +120949.99 & 50 & 50 & 4 & 4 \\
\hline 34 & 212957.874 & +120949.28 & 20 & 40 & 2 & 3 \\
\hline 35 & 212958.732 & +120956.37 & 280 & 90 & 2 & 2 \\
\hline 36 & 212957.302 & +121006.97 & 50 & 80 & 6 & 6 \\
\hline 37 & 212958.086 & +120948.63 & 20 & 60 & 3 & 4 \\
\hline 38 & 212958.621 & +121009.40 & 10 & 30 & 3 & 2 \\
\hline 39 & 212957.802 & +120948.81 & 140 & 40 & 2 & 2 \\
\hline
\end{tabular}

Le Campion et al. (1992a) used measurements of photographic plates covering one to two degrees around M15. Newer catalogues of Aurière \& Cordoni (1981) based on image tube films and the above mentioned HST observations contain only relative positions of stars of the central part of the cluster at arcsecond level. Here we give astrometric positions of stars in the central $3^{\prime} \times 3^{\prime}$ part of M15. Field and cluster are ideal for the calibration of the CCDs observing the planetary satellites. Observations of this kind will be useful for the mutual phenomena of the satellites of Saturn in 1995. Previous observations concerning the mutal phenomena of the satellites of Jupiter (Le Campion et al. 1992b) have shown that a good astrometric calibration is very important.
In this paper we describe the observations and the measurements of the M15 fields, the reduction method and we give the final positions of 1092 stars.

\section{Observations and measurements}

We used the $(D=0.6 \mathrm{~m}, f=9.6 \mathrm{~m})$ Cassegrain telescope of the Observatoire de Bordeaux. The detector was a CCD camera with a Thomson TH 7863 chip, whose dimensions are $384 \times 288$ pixels each of them having a length of 23 microns. This corresponds to a field of $3.4^{\prime} \times 2.5^{\prime}$. We used a two-stage Peltier unit, which cools the CCD down to $-40^{\circ}$. The whole cluster was covered with nine fields, one on the centre and eight around it with a partial 
overlapping. Each field was observed three times with exposure times of 5,10 and 20 seconds. Our data cover a total area of $8^{\prime} \times 5^{\prime}$. The position of each star is given by its photocenter calculated by the software ASTROL (Colas 1991). This software was modified during our reductions. Therefore, two different procedures are used, one for the exposures of 5 and 20 seconds and the other for that of 10 seconds, which were reduced later. In the first case the sky background of a window containing the star was subtracted and the centre of intensity was calculated. In the second case gaussian fitting was used.
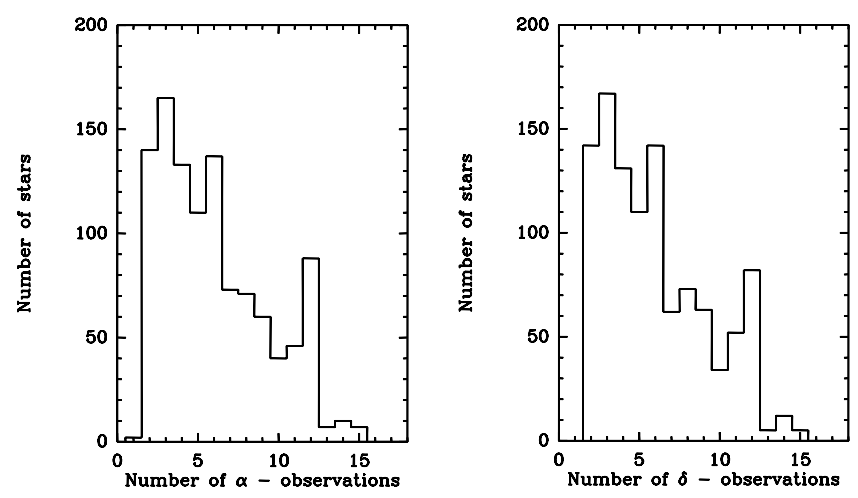

Fig. 1. a) Distribution of the number of observations in right ascension. b) Distribution of the number of observations in declination

\section{Reduction and results}

The reference catalogue used to obtain right ascensions and declinations of our stars and the reduction method are given in Le Campion et al. (1992a). This catalogue was determined with respect to the PPM catalogue, which is a representation of the FK5. Therefore our catalogue is in the FK5 system. However, due to possible systematic errors of the PPM for small fields, we wish to point out, that our positions should not be used for absolute astrometry. For the reduction of the CCD data the same iterative procedure as in Le Campion et al. (1992a) was used. We included plate constants with polynomials up to second order of the rectangular coordinates in $x$ and $y$. In the following, we will denote with 'precision of our positions' the rms of the mean positions determined from the positions of the stars on the different frames. This value of the individual position of each star depends mainly on its magnitude and the number of exposures of the star. The precision of the positions of the stars in overlapping regions of several frames reaches 20 mas, while for the majority of the stars this value amounts to 80 mas. Figures $1 \mathrm{a}$ and $1 \mathrm{~b}$ show the distribution of the number of observations in right ascension and declination of the stars. The data of the catalogue are given in Table 1 for the first 40 stars, the complete catalogue will be available in the
CDS Strasbourg archive. The mean epoch of the catalogue is 1992. Identification charts and their overlays are presented in Figs. 2 to 10. We are aware of the fact, that the use of the charts and the overlays for the centre of the cluster is difficult due to crowding effects. More detailed charts and overlays of the centre are available on request. The distribution of the precisions of our positions in right ascension are given in Fig. 11a and for declination in Fig. 11b.
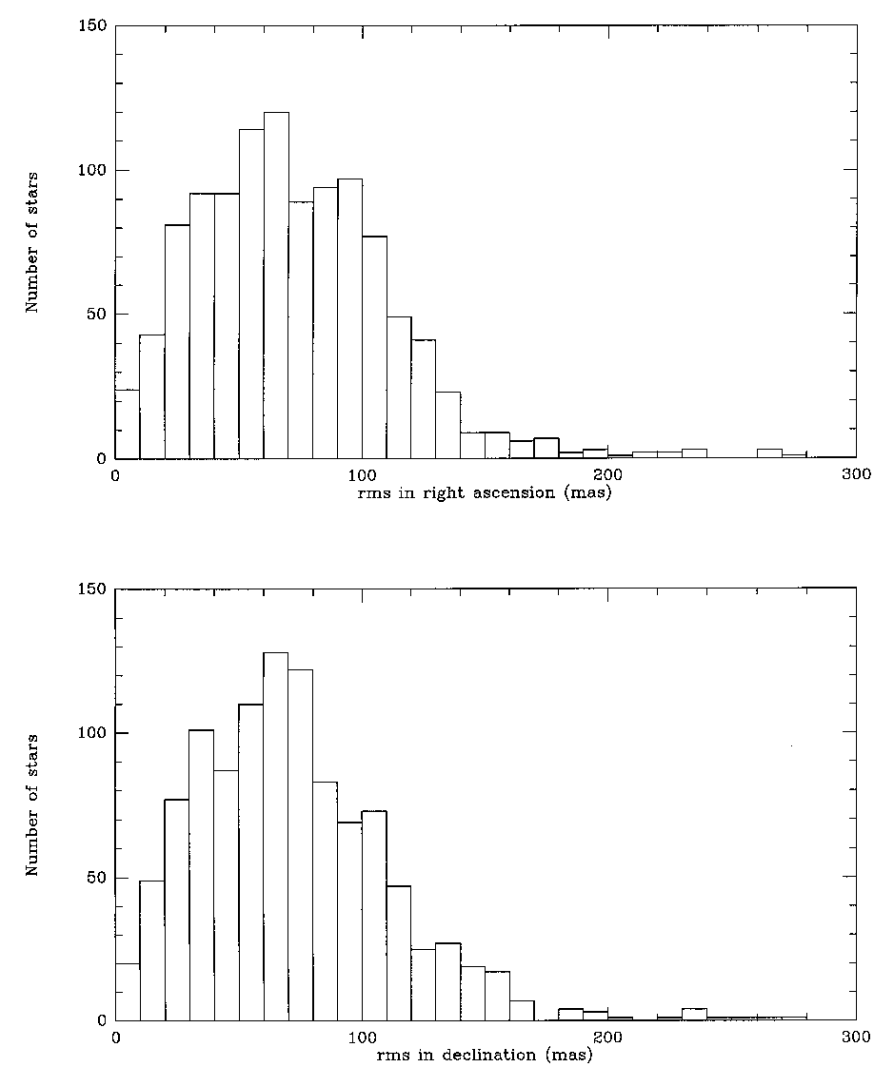

Fig. 11. a) The distribution of the precision of our positions in right ascension. b) The distribution of the precision of our positions in declination

In cases where a large field is available, one may choose from Table 1 only stars with high precision coordinates. As an example, a first use of our catalogue for the calibration of CCD frames for the determination of the distances of the satellites of Saturn showed mean deviations of only 20 mas (Le Campion et al., in preparation).

\section{Comparison with other catalogues}

A comparison of the positions of the 693 stars of our catalogue common to the ones published by Küstner (1921) shows mean differences of 179 mas in right ascension and 181 mas in declination. This comparison includes the proper motion differences between the epoch of the Küstner data (1917) and now, since not all stars in the 
Küstner sample may be cluster stars. In addition, the measurements of the stars in crowded regions on CCD frames, are more difficult to measure on the photographic plates and will lead to systematic differences. Note that the Bonner double refractor, which was used for the Küstner catalogue, has a focal length of only about $5 \mathrm{~m}$. We conclude therefore that a comparison with that catalogue is not suited for a test of our catalogue.

The situation is much better for the catalogue of Buonanno et al. (1983). The focal length of their telescope is about $15 \mathrm{~nm}$ and because their epoch is 1976 , the position differences due to the proper motions will not be as important as for the comparison with the Küstner catalogue. However, the positions in that paper are only relative positions on one standard plate. Moreover, Buonanno et al. (1983) have determined only their post-fit residuals of each star and did not compare the positions of the stars on different plates. A comparison with such data will also include every optical distortion of the telescope. Nevertheless, the comparison with the positions of that catalogue gives for 203 stars in common mean deviations of only 96 mas in right ascension and 94 mas in declination. Assuming that the errors of our catalogue and the Buonanno catalogue are equal, we arrive at 70 mas error for both catalogues, which in view of the large number of our stars seems to be sufficient for the astrometric calibration of CCD frames.

As a further astrometric test we have compared our positions with measurements of plates from the 3.5 meter telescope of the Calar Alto taken in 1982. The comparison was based on data of 144 stars. Excluding the stars Ku284, Ku663 and Ku809 (because of their large proper motions) and using reduction models with polynomials up to third order of the rectangular coordinates on the plate, we found deviations of about 50 mas, which may be taken as an upper limit of the precision of the positions of our stars used in this comparison.

\section{Summary}

We have established a catalogue of 1092 stars in the central region $8^{\prime} \times 6^{\prime}$ of the globular cluster M15. Comparison with other catalogues and measurements show mean differences below 100 mas which indicate a precision of our catalogue between 50 and 70 mas, depending on the ratio of the high accuracy stars from the overlapping zones and 'normal' stars. For the future, we are planning to include further material which allows also the determination of the proper motions of the stars.

Acknowledgements. We thank U. Hopp (Heidelberg) for lending us the Calar Alto plates. We are also indebted to H.-J. Tucholke for scanning the Calar Alto plates at the PDS in Münster.

\section{References}

Aurière M., Cordoni J.-P., 1981, A\&AS 46, 347

Aurière M., Le Fèvre O., Terzan A., 1984, A\&A 138, 415

Battistini P., Bregoli G., Fusi Pecci F., Lolli M., 1985, A\&AS 61, 487

Buonanno R., Buscema G., Corsi C.E., Iannicola G., Fusi Pecci F., 1983, A\&AS 51, 83

Colas F., 1991, PhD thesis, University of Paris

De Marchi G., Paresce F., 1994, ApJ 422, 597

Geffert M., Sinachopoulos D., Guibert J., 1993a, Very wide "visual double stars" for the astrometric calibration of CCD's. In: McAlister H.A., Hartkopf W.I. (eds) Astron. Soc. Pac. Conf. Ser. 32, Complementary approaches to double and multiple star research, San Francisco, p. 137

Geffert M., Colin J., Le Campion J.F., Odenkirchen M., 1993b, AJ 106, 168

Geffert M., Aurière M., Requième Y., Mazurier J.-M., 1994, A\&A 282, 989

Küstner F. 1921, Veröff. Stern. Bonn 15

Lauer, T.R., Holtzman J.A., Faber S.M., et al., 1991, ApJ 369, L45

Le Campion J.F., Geffert M., Dulou, M.R., Colin J., 1992a, A\&AS 95, 233

Le Campion J.F., Montignac G., Chauvet F., et al., 1992b, A\&A 266, 568

Monet D.G., Dahn C.C, 1983, AJ 88, 1489

Prince T.A., Anderson S.B., Kulkarni S.R., Wolszczan W., 1991, ApJ 347, L41

Requième Y., Montignac G., Le Campion J.-F., et al., 1995, First results of the Bordeaux meridian circle equipped with a CCD detector. In: E. Hog, P.K. Seidelmann (eds.) Proc. IAU Symp. 166, Astronomical and astrophysical objectives of sub-milliarcsecond optical astrometry. Kluwer, Dordrecht, p. 358

Sinachopoulos D.,Seggewiß W., 1990, A\&AS 83, 245

Sinachopoulos D., Oblak E., Geffert M., et al., 1994, Astrometric standard fields for CCD observations of double stars. In: MacGillivray H.T. et al. (eds.) Proc. IAU Symp. 161, Astronomy from wide field imaging. Kluwer, Dordrecht, p. 291 Stetson P.B., 1994, PASP 106, 250

Taylor J.H., Manchester R.N., Lyne A.G., 1993, ApJS 88, 529 Yanny B., Guhathakurta P., Bahcall J.N., Schneider D.P., 1994, AJ 107, 1745 

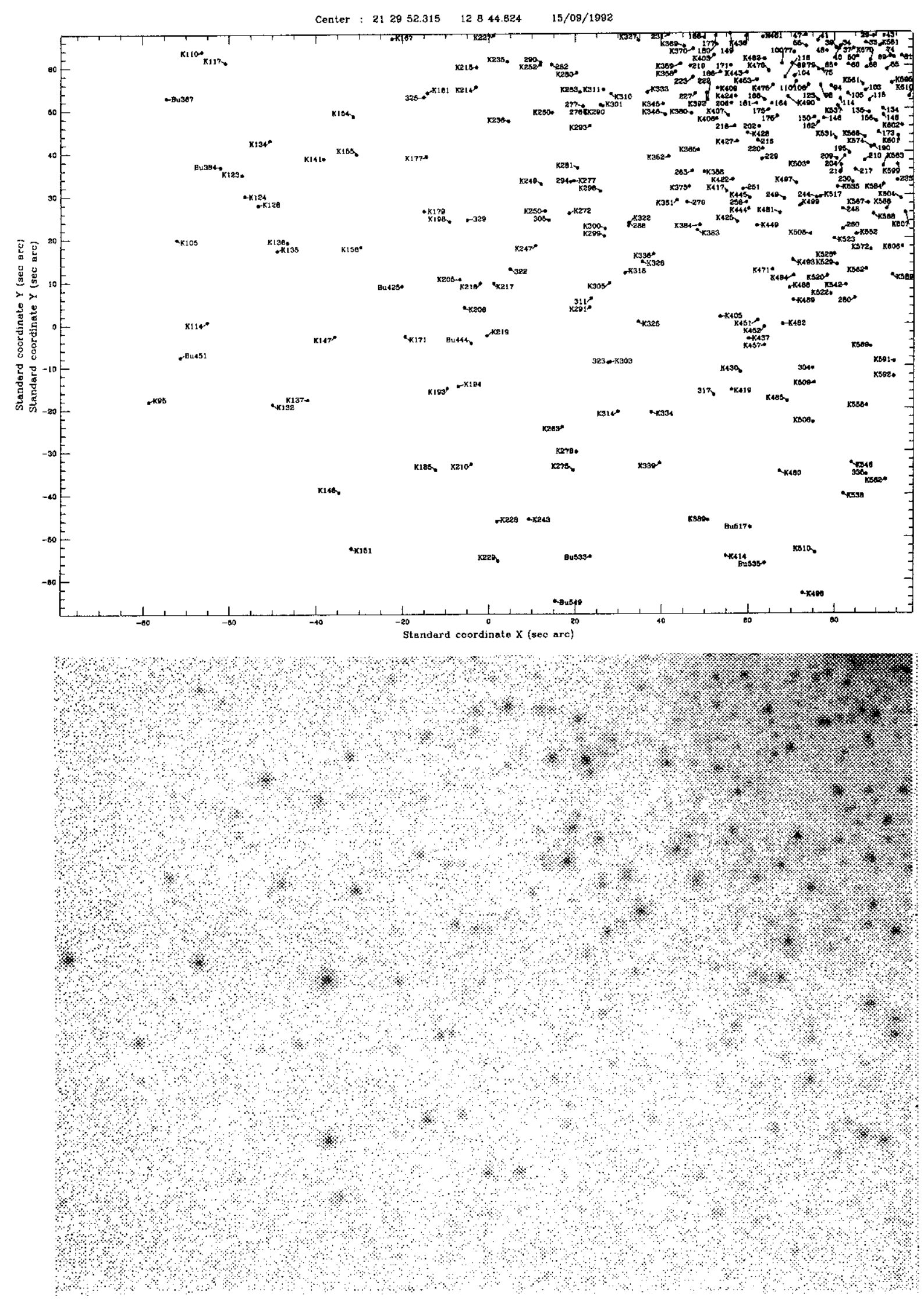

Fig. 2. Overlay and identification chart for field 1 of our catalogue 

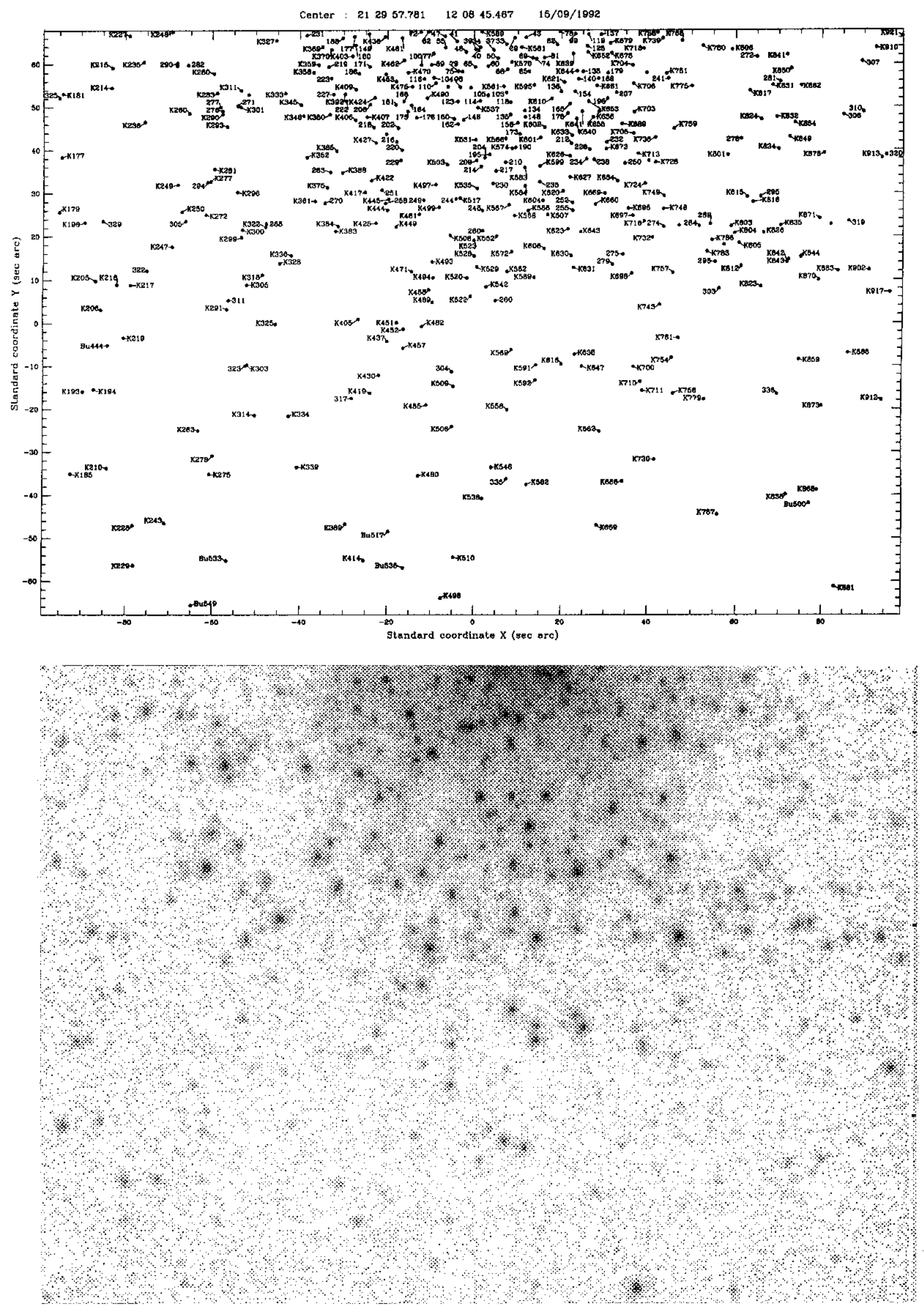

Fig. 3. Overlay and identification chart for field 2 of our catalogue 

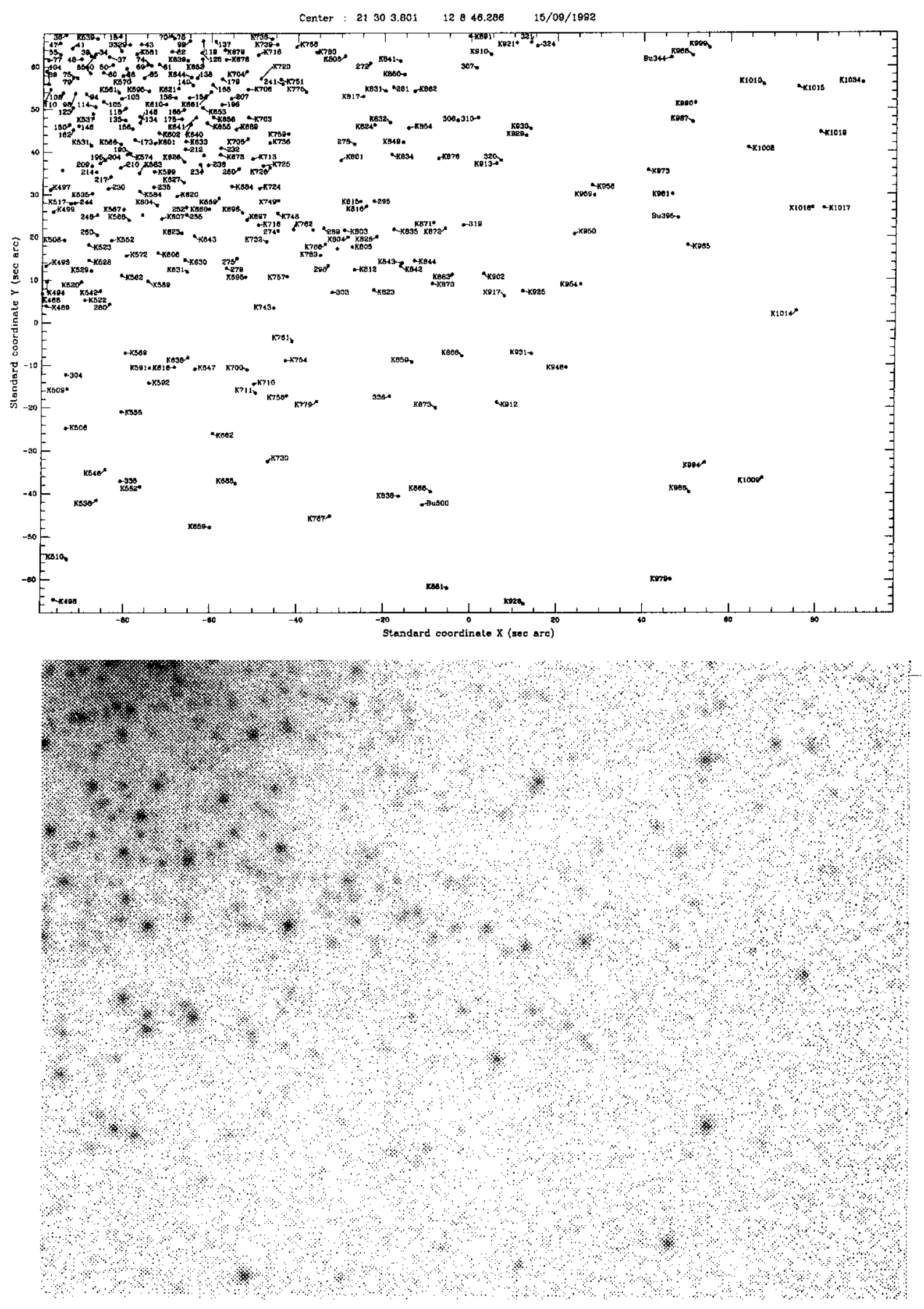

Fig. 4. Overlay and identification chart for field 3 of our catalogue 

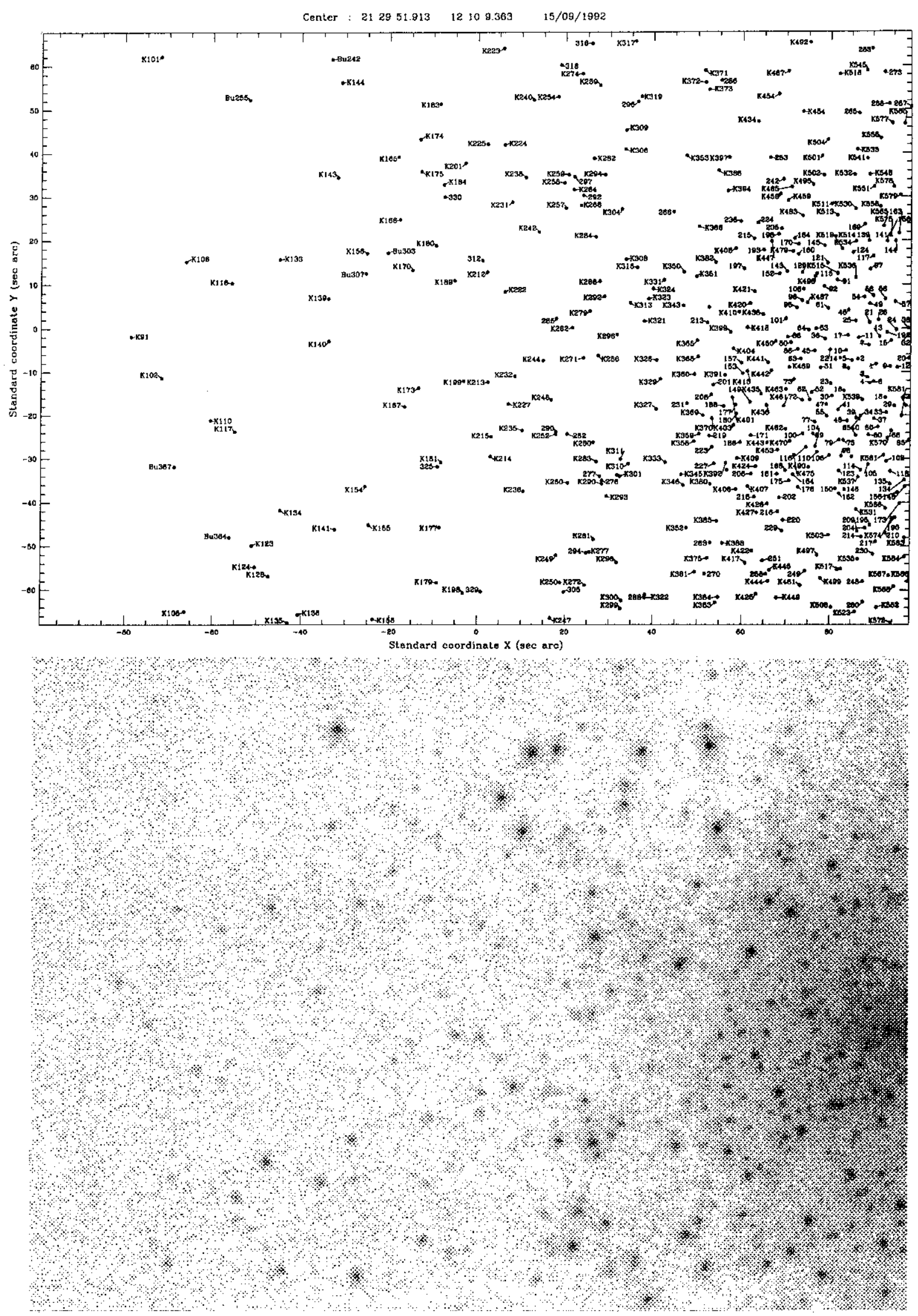

Fig. 5. Overlay and identification chart for field 4 of our catalogue 

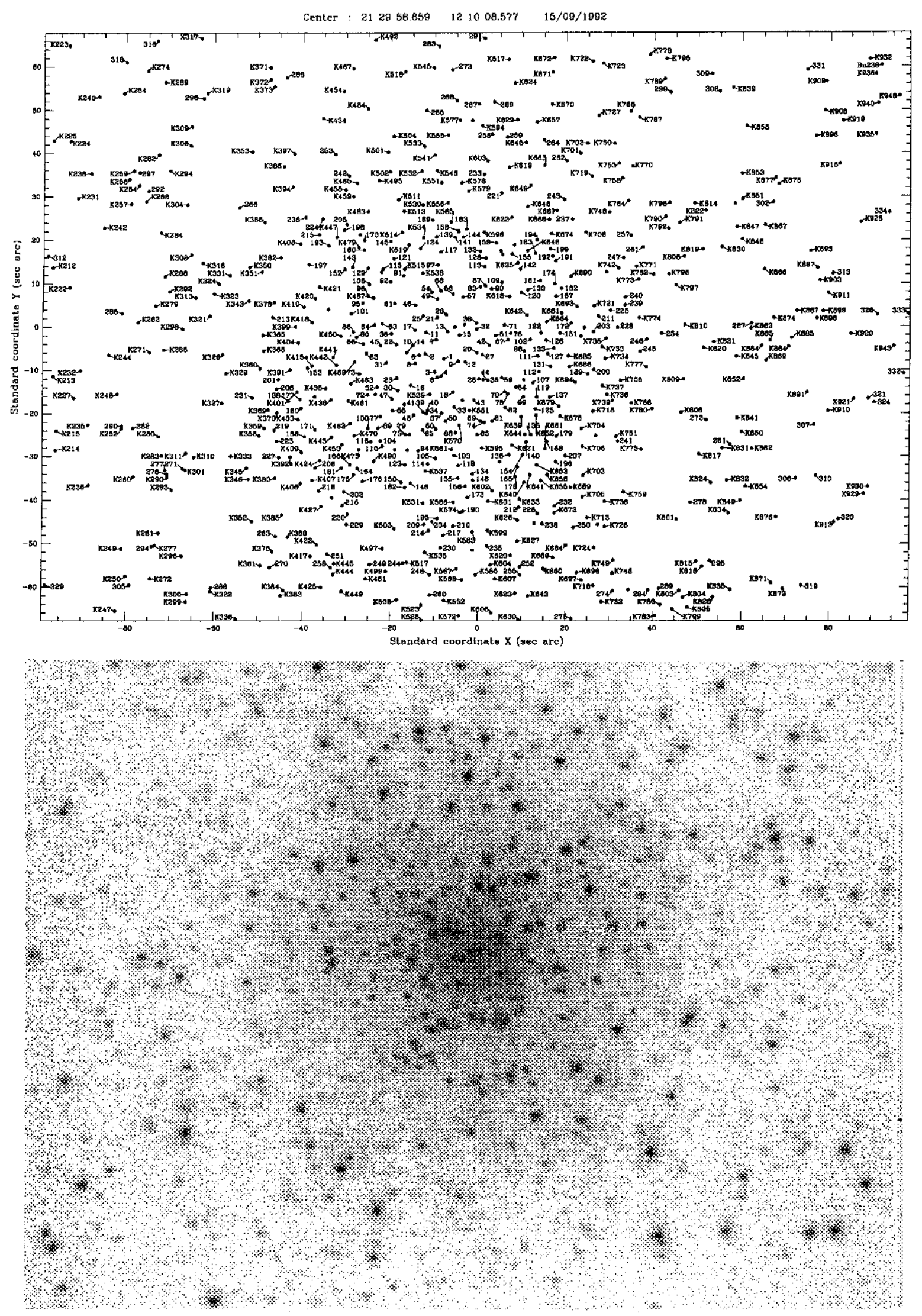

Fig. 6. Overlay and identification chart for field 5 of our catalogue 

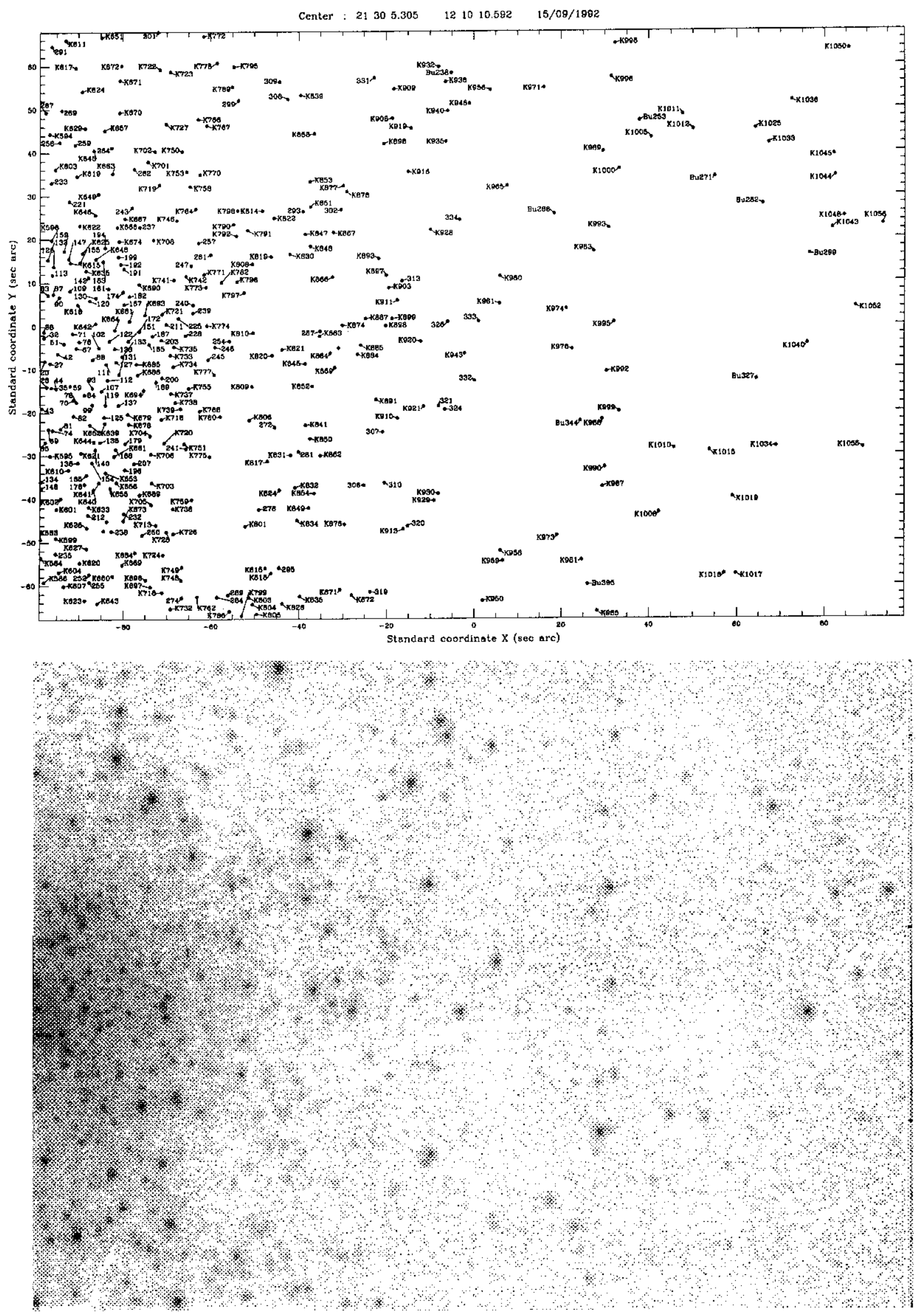

Fig. 7. Overlay and identification chart for field 6 of our catalogue 

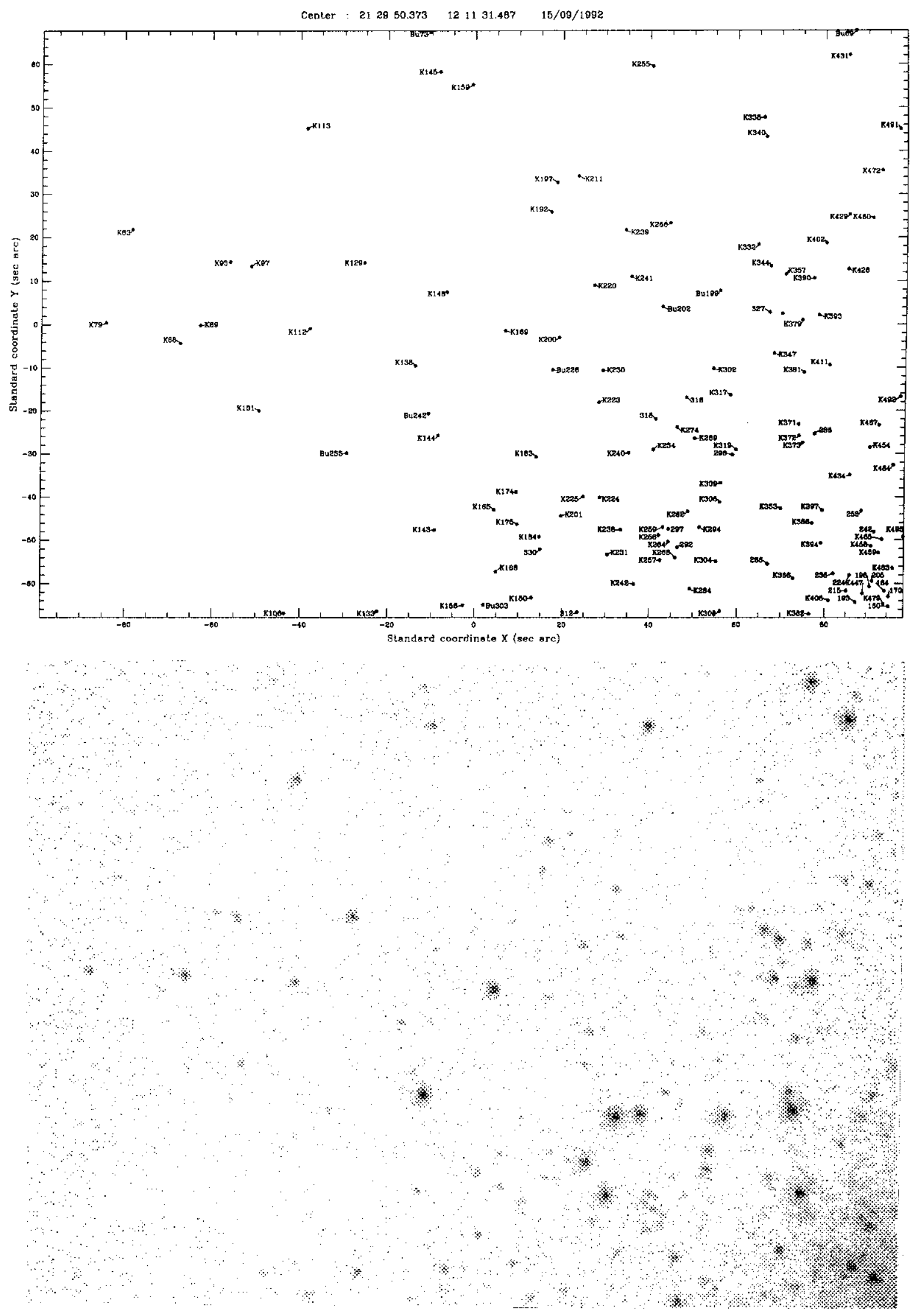

Fig. 8. Overlay and identification chart for field 7 of our catalogue 

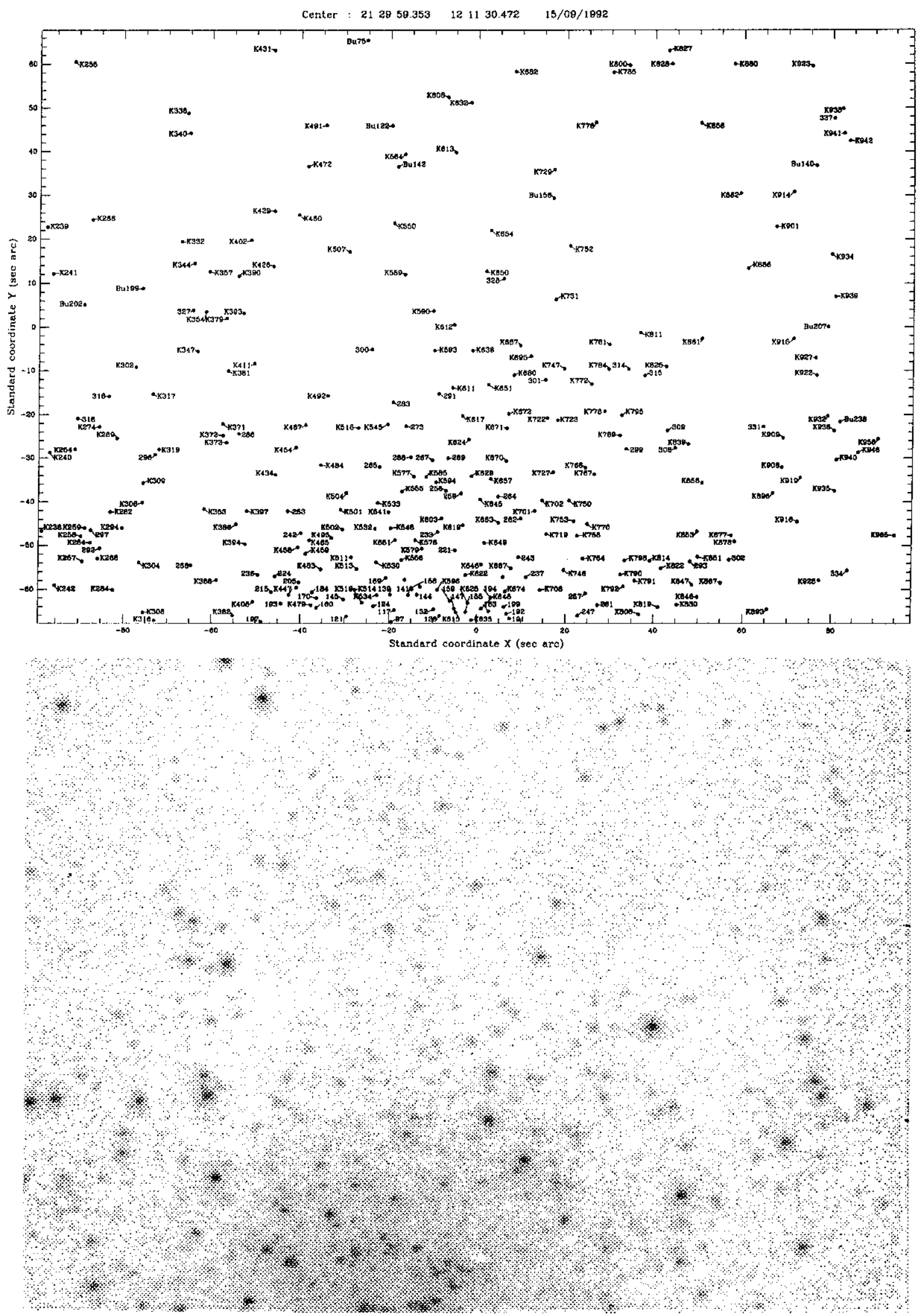

Fig. 9. Overlay and identification chart for field 8 of our catalogue 

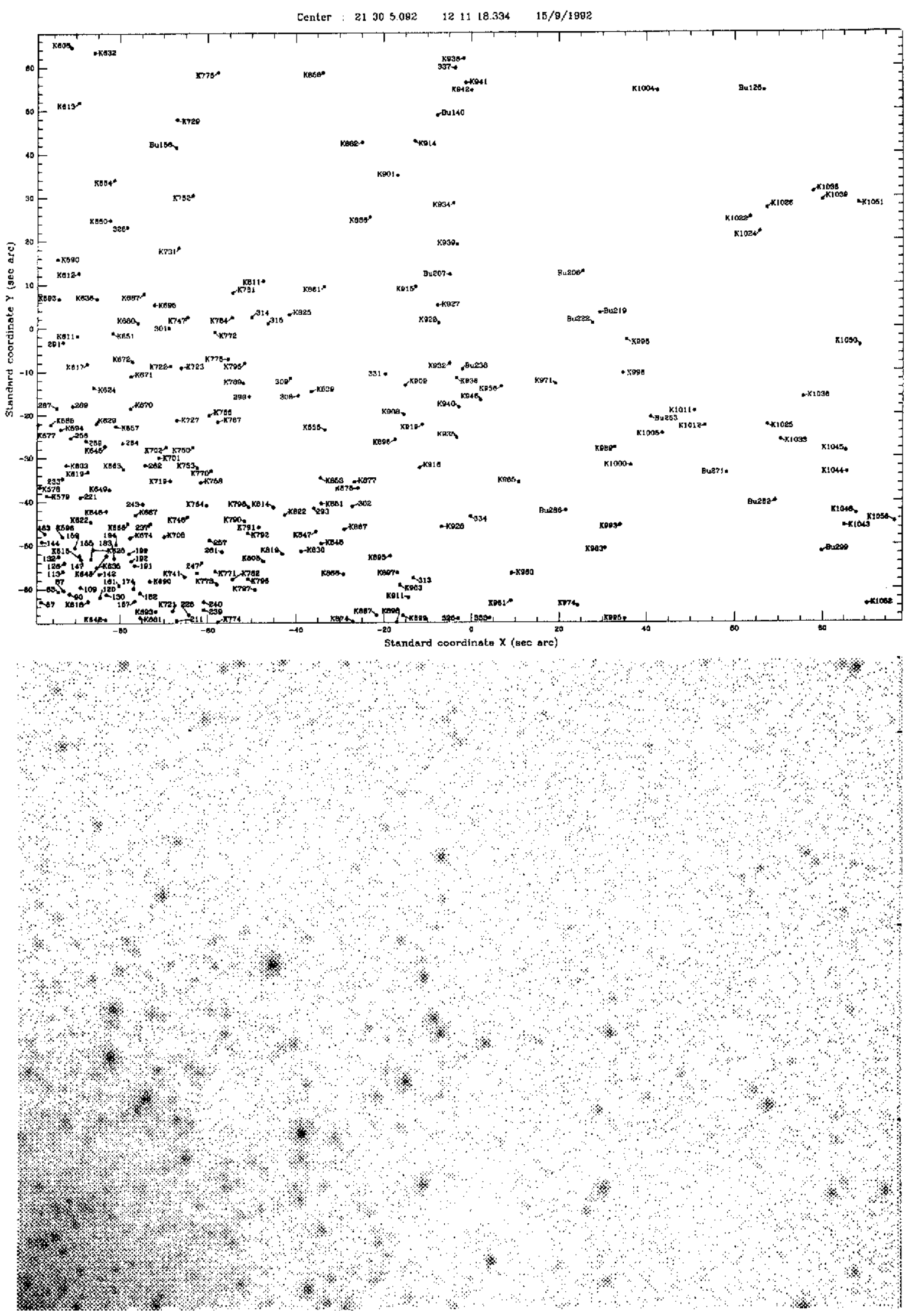

Fig. 10. Overlay and identification chart for field 9 of our catalogue 